\title{
Superposition as a logical glue
}

\author{
Andrea Asperti \\ Department of Computer Science, University of Bologna \\ asperti@cs.unibo.it
}

\author{
Enrico Tassi \\ Microsoft Research-INRIA Joint Centre \\ enrico.tassi@inria.fr
}

\begin{abstract}
The typical mathematical language systematically exploits notational and logical abuses whose resolution requires not just the knowledge of domain specific notation and conventions, but not trivial skills in the given mathematical discipline. A large part of this background knowledge is expressed in form of equalities and isomorphisms, allowing mathematicians to freely move between different incarnations of the same entity without even mentioning the transformation. Providing ITP-systems with similar capabilities seems to be a major way to improve their intelligence, and to ease the communication between the user and the machine. The present paper discusses our experience of integration of a superposition calculus within the Matita interactive prover. Superposition provides the key ingredient for a "smart" application tactic that allows the user to disregard many tedious details otherwise needed to convince the system that his reasoning step is indeed correct. We also show how this kind of automation, named small scale, can serve as the building block for the more general, large scale, case, allowing a smooth integration of equational reasoning with backward-based proof searching procedures.
\end{abstract}

\section{Introduction}

One usually thinks of Mathematics as a precise discipline, often confusing mathematical rigor with logical formality. In fact, most mathematics is simply too informal to be directly handled by the logical and algebraic means offered by interactive or automated theorem provers. The typical mathematical discourse systematically exploits symbol overloading and notational abuses that can hardly be understood by automatic devices without substantial help from the user side, that is one of the reasons why formal encoding is so expensive and frustrating. The crucial point is that the intrinsic ambiguity of the mathematical vernacular can only be resolved by a sufficiently contextual interpretation, requiring not just the knowledge of its specific notation and conventions, but nontrivial skills in the given mathematical discipline. A large part of this background knowledge is expressed in form of equalities and isomorphisms, allowing a mathematician to freely move between different incarnations (intensions) of the same entity without even mentioning the transformation. Providing ITP-systems with similar capabilities seems to be a major way to improve their intelligence, and to ease the communication between the user and the machine. In the present paper, we discuss our experience of integration of a superposition calculus within the Matita interactive prover, providing in particular a very flexible, "smart" application tactic, and a simple, innovative approach to automation.

The need for a stronger integration between fully automatic (resolution) provers and interactive ones, and more generally for a stronger automation support in proof assistants is a major challenge (see e.g. [13]) and many efforts have been already done in this direction: for instance, KIV has been integrated with the tableau prover $3 T^{A} P$ [1]; HOL has been integrated with various first order provers, such as Gandalf [14] and Metis [15]; Coq has been integrated with Bliksem [6]; Isabelle was first integrated with a purpose-built prover [21] and more recently with Vampire [19].

T. Hirschowitz (Ed.): Types for Proofs and Programs 2009 (TYPES 2009). EPTCS 53, 2011, pp. 1-15, doi:10.4204/EPTCS.53.1 (c) A. Asperti and E. Tassi

This work is licensed under the Creative Commons Attribution License. 
We share most of the principles guiding these efforts, and in particular the need to refer to a large library of known lemmas, and the goal to deliver a checkable proof, in conformity with the trusted kernel philosophy (sometimes referred to as De Bruijn principle) inspiring most interactive provers.

However, there are two different uses of automation that have different requirements and possibly deserve different approaches and solutions.

The first one (small scale automation) is to relieve the user from the burden to fill in relatively trivial steps, by automatically completing the missing gaps. This kind of automation must be fast, robust and sufficiently predictable, in the sense that the automation procedure should not miss simple solutions when they exist. In general, in this case, the user is not interested to read back the proof and there is no point in trying to transform it in a human readable format.

The second use of automation (large scale automation) is to really help the user in the process of devising the proof. In spite of all the progresses in this field, a fully automatic approach still looks highly problematic: a more promising approach seems to be that of improving the cooperation between human and machine, and in particular of making a better profit of the machine's combinatorial capabilities. At present, "interaction" in ITP systems is essentially restricted to a master-slave command execution loop, that frustrates the computational power of machines. A better repartition of the work could consist in leaving to the user the most intelligent tasks, such as identifying the key lemmas, proof principles and intermediate results of interest for the proof, assigning to the system the burden of composing them into a coherent proof (eventually exploiting a huge library of known results). The user has hence the responsibility to cut the search space, while the machine is supposed to automatically and systematically explore it (this was also our guiding idea behind the design of the automation driver of Matita version 0.5.x [3]). Another recent system close to this conception is $\Omega$ mega [5]: in this case the user drives the search by means of proof plans, invoking external reasoners to fullfill them.

For large scale automation, we could bear to run time consuming jobs, possibly working for hours in the background. The result is completely unpredictable, and probably unstable. The system should produce a proof in a format as readable as possible, since the user is surely interested to inspect it himself, apart from pasting it into the proof script (if proof searching is expensive we probably wish to avoid running it over and over every time we recompile the script, or at least to have the possibility to choose between these two alternatives). The heuristic, unstable nature of complex automation procedures, combined with the verbose nature of fully formal proofs, naturally suggest the idea of producing "proof traces" as a compact, readable and reproducible output of automation devices.

This is the point where the two kinds of automation recombine together, since the execution of proof traces precisely requires small scale automation capabilities. Our point is that a good part of these capabilities are fulfilled by reasoning up to equalities, providing the connective glue that constitutes most of that background knowledge tacitly, almost unconsciously used in the typical mathematical reasoning. Superposition provides a natural support for reasoning modulo a congruence on propositions, implementing ideas similar to [10], and providing a flexible and powerful tool for small scale automation.

One of the components of the Matita interactive theorem prover is a state of the art, first order, untyped superposition algorithm, able to compete with the best tools currently available: in particular, our system scored in fourth position in the unit equality division at the 22nd CADE ATP System Competition, beating a glorious system such as Otter, and being awarded as the best new entrant tool [25]. Note in particular that Matita is entirely written in a functional language (OCaml), while most ATP system (with the relevant exception of Metis, that was however beaten by Matita) are written in imperative code.

In this paper, we shall provide a theoretical and architectural description - as self contained as possible - of this tool (Section 2); hence we shall discuss its integration with Matita (Section 3), and show some of its applications, mostly aimed to improve the intelligence of commands (smart application, Section 4) 
and the overall automation of the system (Section 5).

\section{Superposition}

Techniques for equational reasoning are a key component in many automated theorem provers and interactive proof and verification systems $[4,20,7]$. The main deductive mechanism is a completion technique [16] attempting to transform a given set of equations into a confluent rewriting system so that any two terms are equal if and only if they have identical normal forms. Not every equational theory can be presented as such a confluent rewriting system, but you may progressively approximate it by means of a refutationally complete method called ordered completion. The deductive inference rule used in completion procedures is superposition which consists of first unifying one side of one equation with a subterm of another, and hence rewriting it with the other side; the selection of the two terms to be unified is guided by a given term ordering, which imposes certain restrictions on inferences, with the major benefit to prune the search space. All results in this section are known, and we only report them for the sake of completeness.

\subsection{Preliminaries}

Let $\mathscr{F}$ bet a countable alphabet of functional symbols, and $\mathscr{V}$ a countable alphabet of variables. We denote with $\mathscr{T}(\mathscr{F}, \mathscr{V})$ the set of terms over $F$ with variables in $V$. A term $t \in \mathscr{T}(\mathscr{F}, \mathscr{V})$ is either a 0 -arity element of $\mathscr{F}$ (constant), an element of $\mathscr{V}$ (variable), or an expression of the form $f\left(t_{1}, \ldots, t_{n}\right)$ where $f$ is a element of $\mathscr{F}$ of arity $n$ and $t_{1}, \ldots, t_{n}$ are terms.

Let $s$ and $r$ be two terms. $\left.s\right|_{p}$ denotes the subterm of $s$ at position $p$ and $s[r]_{p}$ denotes the term $s$ where the subterm at position $p$ has been replaced by $r$.

A substitution is a mapping from variables to terms. Two terms $s$ and $t$ are unifiable if there exists a substitution $\sigma$ such that $s \sigma=t \sigma$. Moreover, in the previous case, $\sigma$ is called a most general unifier (mgu) of $s$ and $t$ if for all substitution $\theta$ such that $s \theta=t \theta$, there exists a substitution $\tau$ which satisfies $\theta=\tau \circ \sigma$.

A literal is either an abstract predicate (represented by a term), or an equality between two terms. A clause $\Gamma \vdash \Delta$ is a pair of multisets of literals: the negative literals $\Gamma$, and the positive ones $\Delta$. If $\Gamma=\emptyset$ (resp. $\Delta=\emptyset$ ), the clause is said to be positive (resp. negative). A Horn clause is a clause with at most one positive literal. A unit clause is a clause composed of a single literal. A unit equality is a unit clause where the literal is an equality.

\subsection{Term orderings and Inference rules}

A strict ordering $\prec$ over $\mathscr{T}(\mathscr{F}, \mathscr{V})$ is a transitive and irreflexive (possibly partial) binary relation. An ordering is stable under substitution if $s \prec t$ implies $s \sigma \prec t \sigma$ for all terms $t, s$ and substitutions $\sigma$. A well founded monotonic ordering stable under substitution is called reduction ordering (see [8]).

The intuition behind the use of reduction orderings for limiting the combinatorial explosion of the number of equations during inference, is to only rewrite big terms to smaller ones.

superposition left This rule defines backward reasoning steps. The equational fact $l=r$ is combined with the goal $t_{1}=t_{2}$ obtaining a the goal $\left(t_{1}[r]_{p}=t_{2}\right) \sigma$.

$$
\frac{\vdash l=r \quad t_{1}=t_{2} \vdash}{\left(t_{1}[r]_{p}=t_{2} \vdash\right) \sigma}
$$


if $\sigma=m g u\left(l,\left.t_{1}\right|_{p}\right),\left.t_{1}\right|_{p}$ is not a variable, $l \sigma \npreceq r \sigma$ and $t_{1} \sigma \npreceq t_{2} \sigma$;

superposition right This rule defines forward reasoning steps. The two equational facts $l=r$ and $t_{1}=t_{2}$ obtaining a new fact $\left(t_{1}[r]_{p}=t_{2}\right) \sigma$.

$$
\frac{\vdash l=r \quad \vdash t_{1}=t_{2}}{\left(\vdash t_{1}[r]_{p}=t_{2}\right) \sigma}
$$

if $\sigma=m g u\left(l,\left.t_{1}\right|_{p}\right),\left.t_{1}\right|_{p}$ is not a variable, $l \sigma \npreceq r \sigma$ and $t_{1} \sigma \npreceq t_{2} \sigma$;

equality resolution This is the rule that ends the proof search.

$$
\frac{t_{1}=t_{2} \vdash}{\square}
$$

if there exists $\sigma=m g u\left(t_{1}, t_{2}\right)$.

\subsection{Simplification rules}

For efficiency reasons, the calculus must be integrated with a few additional optimization rules, the most important one being demodulation ([26]):

subsumption This rule allows to identify and drop clauses that happen to be the instance of more general ones, and are thus superflous.

$$
S \cup\{C, D\} \Rightarrow S \cup\{C\}
$$

if $C$ subsumes $D$, i.e. if there exists a substitution $\sigma$ such that $C \sigma \equiv D$.

tautology elimination This rule eliminates equational facts that are provable with the equality resolution rule and are thus superflous.

$$
S \cup\{\vdash t=t\} \Rightarrow S .
$$

demodulation This rule aims at reducing the size of the clauses involved in the proof search, speeding up all operations whose complexity is determined by the size of the terms involved. The intuitive idea is to consider clauses modulo know equational facts and record only their smaller representative.

$$
S \cup\{\vdash l=r, C\} \Rightarrow S \cup\left\{\vdash l=r, C[r \sigma]_{p}\right\},
$$

if $\left.l \sigma \equiv C\right|_{p}$ and $l \sigma \succ r \sigma$.

\subsection{The main algorithm}

To avoid combining the same clauses twice, it is convenient to keep them in two sets, that are traditionally called active and passive. The general invariant is that clauses in the active sets have been already composed together in all possible ways. A step consists in selecting some clauses from the passive set, add them to the active set, compose them with the current active set - and thus with themselves (inference), and finally add the newly generated clauses to the passive set (possibly after a simplification). A natural strategy would consist in selecting the whole passive set at each iteration, realizing a sort of breadthfirst strategy. The advantage of this strategy is that it is very predictable, and hence particularly easy to debug. Unfortunately the number of new equations generated at each step grows extremely fast, 
practically preventing to iterate the main loop more than a few steps. To avoid this problem, the opposite solution is usually adopted, consisting in selecting just one passive equation at each step. The equation is selected according to suitable heuristics (size, goal similarity, and so on), usually comprising some fairness criterion to ensure completeness (we must ensure that any passive equation will be selected, soon or later). This approach is called the given-clause algorithm (figure 1), and it is the procedure used

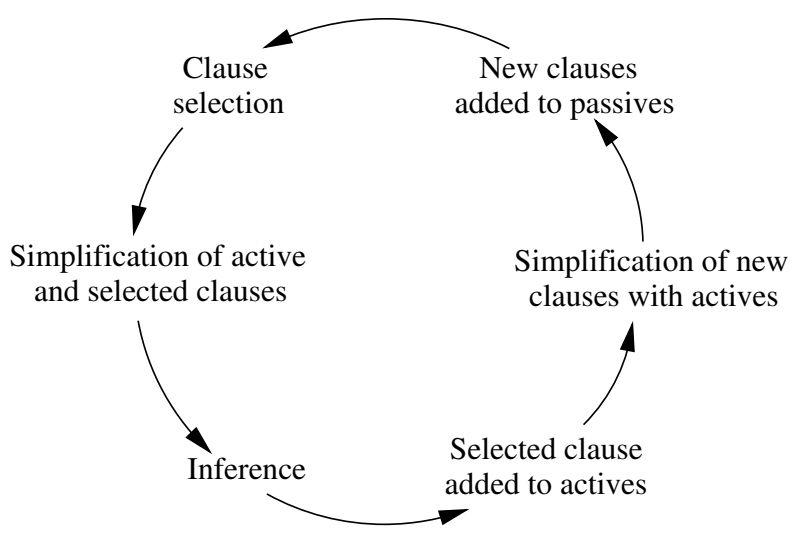

Figure 1: given-clause algorithm

(with some variations) by all modern theorem provers (see e.g. [22]). The advantage of this method is that the passive set grows much slower, allowing a more focused and deeper inspection of the search space. The drawback is that the algorithm becomes extremely sensitive to the selection heuristic, leading to more unpredictable behaviour.

In order to get a high performance tool, the given clause algorithm has to be tuned and optimized in several ways. The critical areas are:

- Data structures and code optimization

- Orderings used to orientate rewriting rules

- Selection strategy

- Demodulation

We are currently using relatively simple data structures (discrimination trees [17]) for term indexing, but we plan to exploit in the near future more specific data structures (such as substitution [12] or context trees [11]).

On complex problems (e.g. problems in the TPTP library with rating greater then 0.30) the choice of a good ordering for inference rules is of critical importance. As we already mentioned, we have implemented several orderings, comprising standard Knuth-Bendix (KBO), non recursive Knuth-Bendix (NRKBO), lexicographic path ordering (LPO) and recursive path ordering (RPO). The best suited ordering heavily depends on the kind of problem, and is hard to predict ${ }^{1}$.

Luckily, on simpler problems (of the kind required for small scale automation) the given-clause algorithm is less sensitive to the term-ordering, and any of them usually produce a solution in a reasonable amount of time.

\footnotetext{
${ }^{1}$ Our approach to the CADE ATP System Competition was to run in parallel different processes with different orderings.
} 
The selection strategy currently implemented by Matita is a based on combination of age and weight. The weight is a positive integer that provides an estimation of the "complexity" of the clause, and is tightly related to the number of occurrences of symbols in it.

Another important issue for performance is demodulation: the given clause algorithm spends most of its time (up to 80\%) in simplification, hence any improvement in this part of the code has a deep impact on performance. However, while reductions strategies, sharing issues and abstract machines have been extensively investigated for lambda calculus (and in general for left linear systems) less is known for general first order rewriting systems. In particular, while an innermost (eager) reduction strategy seem to work generally better than an outermost one (especially when combined with lexicographic path ordering), one could easily create examples showing an opposite behaviour (even supposing to always reduce needed redexes).

Although we did not want to focus too much on developing specific heuristics, two widespread techniques, not yet implemented, would still be of great interest. The first one is Limited Resource Strategy [23], which basically allows the procedure to skip some inference steps if the resulting clauses are unlikely to be processed, because of a lack of time or memory. The other promising technique is indexing modulo associativity and commutativity [9], which is often heavily used when working on algebraic structures.

\section{Integrating superposition with Matita}

\subsection{Library management}

A simple possibility for integrating superposition with Matita is simply to solve goals assuming as initial passive set all equational facts in the library (plus the equations in the local context). The main drawback of this approach is that passive equations would be selected slowly, and in a quite repetitive way every time a new problem is met. In fact, superposition right, as any forward operation, only concern facts, and apart from the local hypothesis, most of these facts are known in advance. This suggest that, in ITP systems, forward operations should be processed, as much as possible, off line; but then we have to face the dual problem, namely to avoid an unnecessary proliferation of results, polluting the library (and the memory) with trivialities. The compromise adopted in Matita was suggested by the observation that, in a given-clause algorithm, selection is a conspicuous operation requiring an intelligent choice; but all theorems in the library are indeed already a "selected" subset (otherwise, there would be no point to record them). In other words, the idea is to use the unit equalities in the library not as initial passive set, but as the active one. This means that every time a new equality is added to the library it also goes through one cycle of the given-clause algorithm as if it was the newly selected passive equation: it is composed (after simplification) with all existing active equations (that is, up to simplifications, all previously proved equalities), and the newly created equations are added to the passive list $^{2}$ This way, we have a natural, simple but traceable syntax to drive the saturation process: it is enough to explicitly list in the library the selected equation. At the same time, this approach reduces the verbosity of the library, since trivial results generated by superposition in the passive list may be used without the need to declare (and name) them explicitly.

\footnotetext{
${ }^{2}$ This approach is particularly important in view of the fact that, typically, the passive set is not even used for demodulation.
} 


\subsection{Input/Output}

The communication between Matita and the superposition tool is not precise. As we already said, our superposition algorithm is first order and untyped; instead of attempting a complex encoding of the Calculus of Inductive Constructions (CIC) in first order logic (that is the approach adopted e.g. in [18]), we prefer to use a naive, but efficient translation, possibly losing information. We shall then try to automatically reconstruct the missing information during proof reconstruction, exploiting the sophisticated inference capability of the Matita refiner [2]. As a consequence, automation is a best effort service: not only it may obviously fail to produce a proof, but sometimes it could produce an argument that the system is not able to refine correctly (independently from the fact if the delivered proof was "correct" or less).

Although there is no particular problem to implement a typed superposition algorithm, or even embedding types as first order terms (in more or less naive ways, according to the way we wish to take convertibility into account), for performance reasons we decided to work with completely untyped terms. In particular, equations $r={ }_{T} s$ of the calculus of constructions are translated to first order equations by merely following the applicative structure of $r$ and $s$, and translating any other subterm into an opaque constant. The type $T$ of the equation is recorded, but we are not supposed to be able to compute types for subterms.

Since all equations are combined together via superposition rules, there is a (modest) risk of producing "ill-typed" terms. Consider for instance the superposition left rule (the reasoning is similar for the other rules)

$$
\frac{\vdash l=r \quad t_{1}=t_{2} \vdash}{\left(t_{1}[r]_{p}=t_{2} \vdash\right) \sigma}
$$

where $\sigma=m g u\left(l,\left.t_{1}\right|_{p}\right)$ and $l \sigma \npreceq r \sigma$. The risk is that $\left.t_{1}\right|_{p}$ has a different type from $l$, hence resulting into an illegal rewriting step. Note however that $l$ and $r$ are usually rigid terms, whose type is uniquely determined by the outermost symbol. Moreover, $\left.t_{1}\right|_{p}$ cannot be a variable, hence they must share this outermost symbol. If $l$ is not rigid, it is usually a variable $x$ and if $x \in r$ (like e.g. in $x=x+0$ ) we have (in most orderings) $l \preceq r$ that again rules out rewriting in the wrong direction.

This leads us to the following notion of admissibility. We say that an applicative term $f\left(x_{1}, \ldots, x_{n}\right)$ is implicitly typed if its type is uniquely determined by the type of $f$. We say that an equation $l=r$ is admissible if both $l$ and $r$ are implicitly typed, or $l \preceq r$ and $r$ is implicitly typed. If an equation is not admissible, we forbid to take it into account for superposition.

\section{3 (Re)construction of the proof term}

Reading back a superposition proof inside any interactive prover is a relatively simple operation (just requiring rewriting), and one of the reasons for sticking to this fragment. In the superposition module, each proof step in encoded as a tuple

Step of rule $*$ int $*$ int $*$ direction $*$ position $*$ substitution

where rule is the kind of rule which has been applied, the two integers are the two $i d^{\prime} s$ of the composing equations (referring to a "bag" of unit clauses), direction is the direction the second equation is applied to the first one, position is a path inside the rewritten term and finally substitution is the mgu required for the rewriting step. The proof has the shape depicted in Figure 2, where all superposition left steps are on the rightmost spine leading from the Goal to the empty clause. Superpostion right steps are forward rewriting operations and their translation is straightforward; on the other side, superposition left steps must be reverted in order to build a direct proof of the (suitably instantiated) goal from its refutation. 


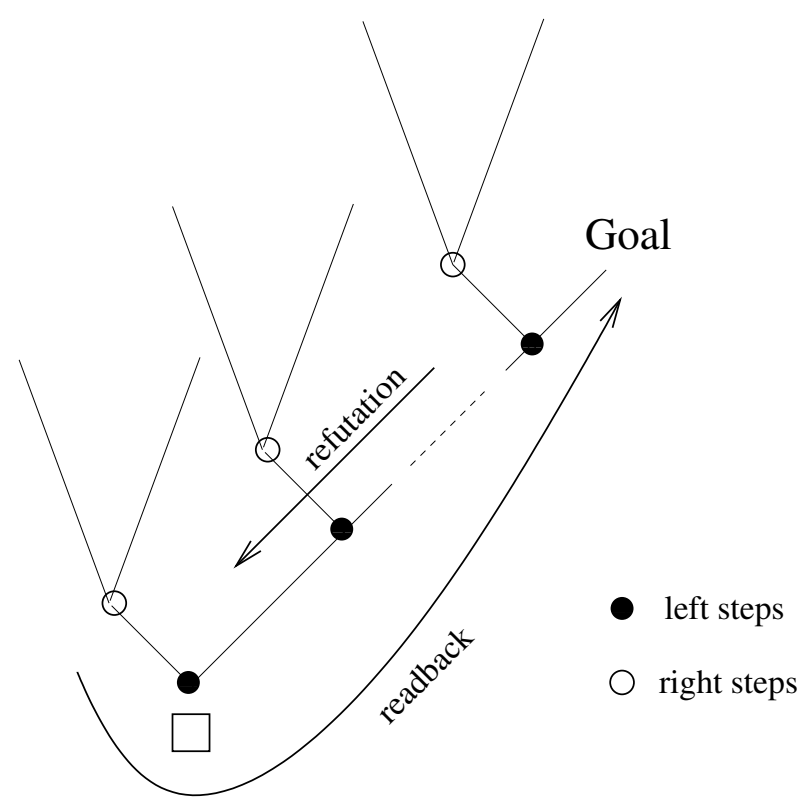

Figure 2: given-clause algorithm

Formally, let's call eq_ind the higher order rewriting step

$$
\text { eq_ind }: \forall A: \text { TYPE. } \forall x: A . \forall P: A \rightarrow \text { Prop.P } x \rightarrow \forall y: A . x=y \rightarrow P y
$$

Let us consider a superposition right step

$$
\frac{\vdash l={ }_{A} r \quad \vdash t={ }_{B} s}{\vdash t[r]_{p} \sigma={ }_{B} s \sigma}
$$

If $h: l={ }_{A} r$ and $k: t={ }_{B} s$ then

$$
\text { eq_ind A } l \sigma\left(\lambda x: A . t[x]_{p}={ }_{B} s\right) \sigma k \sigma r \sigma h \sigma: t[r]_{p} \sigma={ }_{B} s \sigma
$$

Conversely, given a superposition left step

$$
\frac{\vdash l={ }_{A} r \quad \alpha: t={ }_{B} s \vdash}{t[r]_{p} \sigma={ }_{B} s \sigma \vdash}
$$

if $h: r={ }_{A} l$ and $k: t[r]_{p} \sigma={ }_{B} s \sigma$ then

$$
\text { eq_ind } A \operatorname{r} \sigma\left(\lambda x: A \cdot t[x]_{p}={ }_{B} s\right) \sigma k l \sigma h \sigma: t \sigma={ }_{B} s \sigma
$$

To generate a CIC proof term, clauses are topologically sorted w.r.t. their dependendices (to respect scoping), their free variables are explicitly quantified, and nested let-in patterns are used to build the proof. A delicate point of the translation is closing each clause w.r.t. its free variables, since we should infer a type for them. The simplest solution is to generate so called "implicit" arguments leaving to the Matita refiner [2], the burden of guessing them. For instance, superposing plusC: $x+y=y+x$ with plusA : $x+(y+z) \leftrightarrows(x+y)+z$ at the underlined position and in the given direction gives rise to the following piece of code, where question marks stands for implicit arguments: 


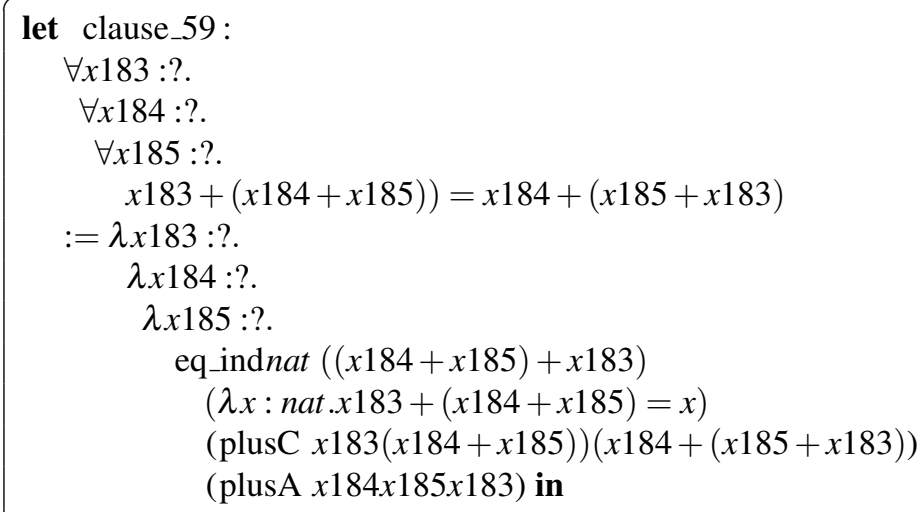

\section{Smart applications}

The first interesting application of superposition (apart its use for solving equational goals), is the implementation of a more flexible application tactic. As a matter of fact, one of the most annoying aspects of formal development is the need of transforming notions to match a given, existing result. As we already said, most of these transformations are completely transparent to the typical mathematical discourse, and we would like to obtain a similar behaviour in interactive provers.

Given a goal $G$ and a theorem t: $\Gamma \rightarrow A$, the goal is to try to match $A$ with $G$ up to the available equational knowledge base, in order to apply $t$. We call it, the smart application of $t$ to $G$.

We use superposition in the most direct way, exploiting on one side the higher-order features of CIC, and on the other the fact that the translation to first order terms does not make any difference between predicates and functions: we simply generate a goal $A=G$ and pass it to the superposition tool (actually, it was precisely this kind of operation that motivated our original interest in superposition). If a proof is found, $G$ is transformed into $A$ by rewriting and $t$ is then normally applied.

Superposition, addressing a typically undecidable problem, can easily diverge, while we would like to have a reasonably fast answer to the smart application invocation, as for any other tactic of the system. We could simply add a timeout, but we prefer to take a different, more predictable approach. As we already said, the overall idea is that superposition right steps - realising the saturation of the equational theory - should be thought of as off line operations. Hence, at run time, we should conceptually work as if we had a confluent rewriting system, and the only operation worth to do is narrowing (that is, left superposition steps). Narrowing too can be undecidable, hence we fix a given number of narrowing operations to apply to each goal (where the new goal instances generated at each step are treated in parallel). The number of narrowing steps can be fixed by the user, but a really small number is usually enough to solve the problem if a solution exists.

Example 1 Suppose we wish to prove that the successor function is $\leq$-reflecting, namely

$$
(*) \quad \forall n, m \cdot S n \leq S m \rightarrow n \leq m
$$

Suppose we already proved that the predecessor function is monotonic:

$$
\text { monotonic_pred }: \forall n, m . n \leq m \rightarrow \text { pred } n \leq \text { pred } m
$$


We would like to merely "apply" the latter to prove the former. Unfortunately, this would not work, since there is no way to match pred $X \leq$ pred $Y$ versus $n \leq m$, unless narrowing the former. By superposing twice with the equation $\forall n$.pred $(S n)=n$ we immediately solve our matching problem via the substitution $\{X:=S n, Y:=S m\}$. Hence, the smart application of monotonic_pred to the goal $n \leq m$ succeeds, opening the new goal $S n \leq S m$ that is the assumption in (*).

Example 2 Let us use the notation $A[B / i]$ to express the substitution of $B$ for the $i$-th free variable in A. The substitution lemma says that for all $k, i$

$$
A[B / i][C / i+k]=A[C / S(k+i)][B[C / k] / i]
$$

(where $S$ is the successor function). The idea is to prove the substitution lemma by structural induction over A. Suppose now A is a binder, e.g. a lambda term $F U N(M)$ where $M$ is the body of the function. The definition of substitution tells us that

$$
F U N(M)[B / i]=F U N(M[B / i+1])
$$

Hence, after normalization and elimination of congruent terms, we are left to prove ${ }^{3}$

$$
(M[B / i+1][C /(k+i)+1]=F u n(M[C / S((k+i)+1)][B[C / k] / i+1]
$$

under the inductive hypothesis

$$
\text { Hind }: \forall j . M[B / i][C / k+j]=M[C / S(k+j)][B[C / k] / j]
$$

It is evident that it is enough to instantiate $j$ with $i+1$ but in order to unify $(k+i)+1$ with $k+?_{j}$ we have to use the associativity law for the sum! Hence the smart application of Hind succeeds where the normal application would fail.

\section{The auto tactic}

By itself, smart application is less interesting than expected. The point is that, compared to the effort of finding the "right" theorem $t$ in the library, the work of transforming the goal to match the conclusion is a boring, but minor task.

What is really interesting, instead, is the possibility to combine smart application with a goal-oriented proof searching technique, to achieve a cheap, simple but surprisingly effective management of equality.

According to our philosophy, forward operations in ITP systems should be performed off line, and explicitly or implicitly recorded in the library (if a forward step is really useful in some context, it is likely to be useful in other, similar contexts as well, hence it is a very good candidate to explicitly appear in the library). For this reason, the Matita automation tool is backward-based (backward operations act on the goal, that is only known at run time), essentially trying to build a proof by a repetitive application of tactics. The proof we are looking for is not in normal form: in fact, the most relevant tactic is application, and the automation tool is supposed to explore the library for all known results matching the current goal. In this respect, automation resembles a prolog-like program, and we use a traditional depth-first strategy (with bounded, user configurable depth) to explore the proof space. The main optimizations ${ }^{4}$ implemented are the following:

\footnotetext{
${ }^{3} \mathrm{We}$ added some artificial parenthesis to the terms to emphasize the left associativity of plus.

${ }^{4}$ All these optimizations destroy the so called procedural interpretation of logic programs, and received very little attention by the logic programming community.
} 
goal clustering a cluster in a set of (conjunctive) goals $\Delta=g_{1}, \ldots, g_{n}$ is a minimal subset closed w.r.t. its free variables: any variable appearing in a goal of the cluster can only appear in other goals of the same cluster. Clusters obviously form a partition of the original set; their interest is that the processing of different clusters can be separated by green cuts;

loop detection if a goal $\Delta$ generates another goal $\Delta^{\prime}$ subsumed by the former, the proof branch can be pruned ${ }^{5}$ (if we find a proof for $\Delta^{\prime}$ it works for $\Delta$ as well - recall that variables in goals are existentially quantified).

We also plan to implement a failure cache (indexed by the failure depth); instead, the advantage of caching successes looks much more questionable (either we pre-compute the whole success set, requiring a different proof searching strategy, or we easily end-up duplicating solutions).

Smart application can be easily integrated in our automated proof searching tactic. Per se, due to the severe constraints imposed on superposition, smart application is not much slower than normal application. The real problem is the brutal explosion in the number of candidates. With normal application, using good data structures for indexing the universe of known results (we use discrimination trees [17]), we are able to retrieve, for each goal, a relatively small number of candidates. In the case of smart application, any theorem predicating something "similar" to the goal is a potential candidate. Our notion of similarity is particularly weak: we look for any theorem whose conclusion shares with the goal (possibly up to reduction) the same top predicate.

Note however that what really matters from the complexity point of view is not the number of candidates which are tried, but the number of them whose application succeeds, giving rise to new branches in the the search tree. Luckily, in general, smart application does not sensibly enlarge the number of applicable theorems, and the overall complexity remains feasible, especially for small depths (3/4).

\subsection{Proof traces}

Since most of the time is spent in searching the right theorems composing the proof, a natural idea is to let the automation tactic return a trace of the proof consisting of all library results used to build the proof. We omit the local assumptions, all equations used by superposition, and to further reduce the verbosity of the trace, we also omit all library facts (i.e. all results with no hypothesis, hence appearing in leaf position inside the proof). The resulting set is passed as an optional "by" argument to the auto tactic.

If the argument is present, the automation tactic would use the set passed as an argument as candidates for smart applications, apart from at depth 0 , where facts in the whole library would be taken into account. Local assumptions are always tried, too.

Using these simple proof traces automation becomes extremely fast, and almost comparable to a fully expanded proof script.

\subsubsection{Example}

This is a relatively complex example borrowed from the Matita standard library (in particular, in a contribution regarding lifting and substitution in DeBrujin notation). The goal to prove is $k \leq n-1$ under the assumption $H: j+k<n$, where $j, k$ and $n$ are natural numbers. The relation $n<m$ is definitionally equivalent to $S n \leq m$ where $S$ is the successor function. Note that the successor function is extensionally equal to (but does not coincide with) the operation of adding 1, in the same way as the predecessor function is extensionally equal to (but does not coincide with) the operation of subtracting 1 . Another

\footnotetext{
${ }^{5}$ At present this is only implemented in case $\Delta^{\prime}$ is a single literal.
} 
delicate point is that the minus operation $x-y$ returns 0 when $y>x$, so $S(x-1)=x$ only if $x>0$.

The solution automatically found by Matita is depicted in Figure 3 (the picture is better understood reading it from the bottom to the top): it first applies the monotonicity of the predecessor function, passing

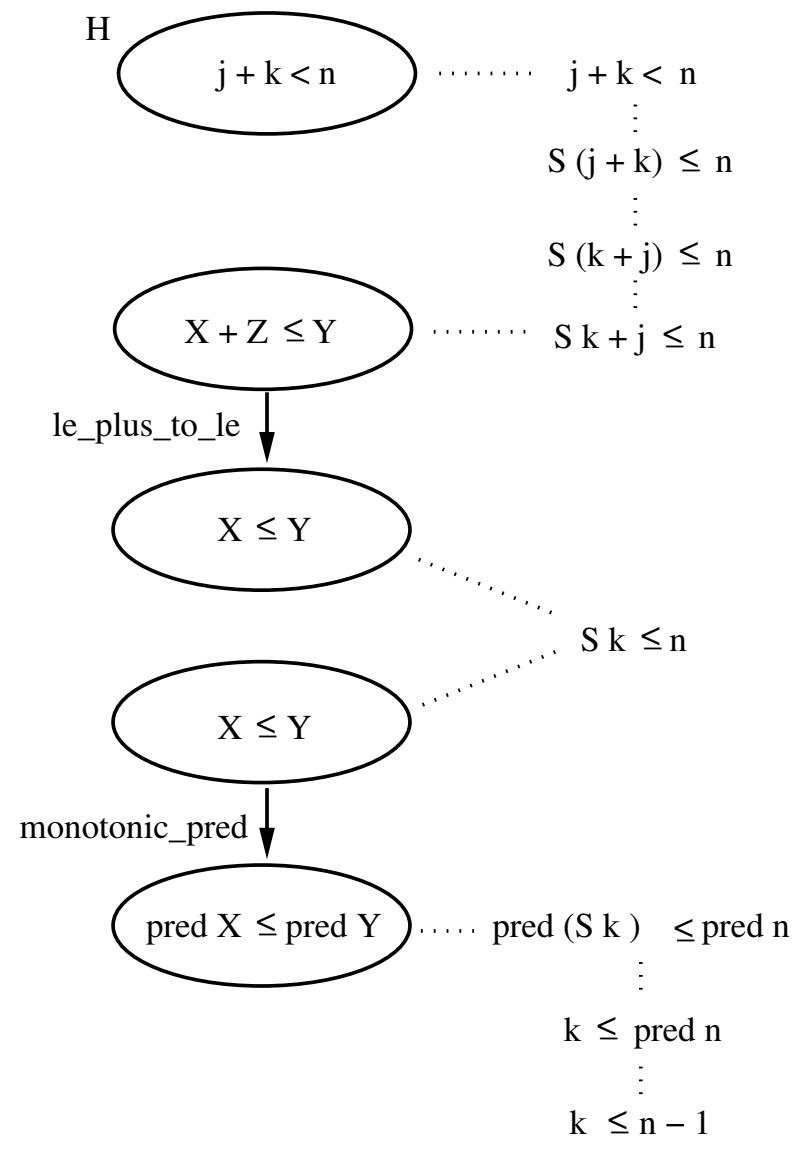

Figure 3: Glueing together lemmas via rewriting

from $k \leq n-1$ to $S k \leq n$; then it applies the lemma

$$
\text { le_plus_to_le }: \forall n, m, a: n+a \leq m \rightarrow n \leq m
$$

obtaining the goal $k+Z \leq n$, and finally applies the hypothesis $H$, instantiating $Z$ with $S j$. In order to do these passages, the system exploits the following equivalences: $k=\operatorname{pred}(S k), n-1=\operatorname{pred} n$, $S k \leq n=k<n, S(j+k)=(S j)+k=k+(S j)$; they are the logical glue permitting to compose together the relevant applicative steps ( le_plus_to_le and monotonic_pred, forming the trace of the proof).

\subsubsection{Some statistics}

We are currently porting the old matita library (containing almost no automation) to the new Matita system. The following table compares the two libraries on a fragment of about one hundred elementary arithmetical theorems. The "size" is the dimension of the gzipped file in bytes. 


\begin{tabular}{c|c|c|c|c|} 
& lines & $\begin{array}{c}\text { size } \\
\text { (whole) }\end{array}$ & $\begin{array}{c}\text { size } \\
\text { (proofs) }\end{array}$ & $\begin{array}{c}\text { compilation } \\
\text { time }\end{array}$ \\
\hline no auto & 1139 & 5753 & $3433(60 \%)$ & $4.6 s$ \\
\hline with auto & 627 & 3788 & $2027(53 \%)$ & $50.4 s$ \\
\hline traces & 627 & 3982 & $2163(54 \%)$ & $5.3 s$ \\
\hline
\end{tabular}

The sensible increase of compilation time in presence of automation was somehow expected: the leading idea of the paper is that we are ready to pay some extra execution time if this can reduce the encoding effort on the user side (provided it does not sensibly slow-down the system reactiveness to user commands in interactive sessions: note that the average execution time per theorem is about 0.35 seconds). Traces seem to provide a natural balance between performance and verbosity.

\section{Conclusions}

In this paper we introduced a general methodology to address the complex problem of automation in interactive provers. The main principles underlying our approach are the following:

1. there is an important distinction to be made between small scale automation, mostly meant to reduce the verbosity of the proof script (resolution of trivial steps, verification of side conditions, automatic inference of missing information, etc.), and large scale automation (problem solving): the problems and requirements in the two cases are different, eventually deserving different approaches and solutions;

2. a major component of small scale automation is the capability to "reason" (apply logical rules and theorems) up to equalities, covering most part of the background knowledge tacitly used in the typical mathematical reasoning as an underlying connective glue between logical steps (see Figure3);

3. large scale automation must return a human readable and system executable proof trace; the trace must be simple, hence its execution will eventually require small scale automation capabilites (independently of the choice of implementing or not large scale automation on top of small scale automation).

The paper also describes the current state of the implementation of this program inside the Matita interactive theorem prover. In particular we presented the architectural design of the superposition tool supporting equational reasoning, and its not so trivial integration inside the Matita Interactive Theorem Prover.

The tool is already highly performant (we scored in fourth position in the unit equality division at the 22nd CADE ATP System Competition), but many improvements can still be done for efficiency. In particular, more specialised data structures for indexes would hopefully give us a chance to scale up with the current best ATPs.

Another interesting research direction is to extend the management of equality to setoid rewriting [24]. Indeed, the current version of the superposition tool merely works with an intensional equality, and it would be interesting to try to figure out how to handle more general binary relations. The main problem is proof reconstruction, but again it looks possible to exploit the sophisticated capabilities of the Matita refiner [2] to automatically check the legality of the rewriting operation (i.e. the monotonicity of the context inside which rewriting has to be performed). 
While we are, at present, reasonably happy of the small scale automation capabilities of Matita, much work is left about large scale automation. Our current approach tries to build large scale automation on top of small scale automation (e.g. substituting application by its smart version); this approach is natural, especially in view of the generation of proof traces but, as we already observed, it is not the only possibility compatible with our methodology and alternative solutions (requiring a tighter integration of small scale automation techniques inside large scale functionalities) are worth to be explored.

Finally, let us remark that proof traces are per se an interesting object worthty of further investigation (and, possibly, standardization), in order to optimize the trade-off between efficiency and verbosity, or to improve interoperability bewteen different systems.

\section{References}

[1] Wolfgang Ahrendt, Bernhard Beckert, Reiner Hähnle, Wolfram Menzel, Wolfgang Reif, Gerhard Schellhorn \& Peter H. Schmitt (1998): Integrating Automated and Interactive Theorem Proving. In Wolfgang Bibel \& Peter H. Schmitt, editors: Automated Deduction - A Basis for Applications. Applied Logic Series, No. 9 II: Systems and Implementation Techniques, Kluwer, Dordrecht, pp. 97-116.

[2] Andrea Asperti, Wilmer Ricciotti, Claudio Sacerdoti Coen \& Enrico Tassi (2009): Hints in Unification. In Stefan Berghofer, Tobias Nipkow, Christian Urban \& Makarius Wenzel, editors: TPHOLs. Lecture Notes in Computer Science 5674, Springer, pp. 84-98, doi:10.1007/978-3-642-03359-9_8.

[3] Andrea Asperti \& Enrico Tassi (2009): An interactive driver for goal directed proof strategies. In: Proc. of User Interfaces for Theorem Provers 2008. Montreal, CA, August 2008. ENTCS 226, pp. 89-105, doi:10.1016/j.entcs.2008.12.099.

[4] Leo Bachmair \& Harald Ganzinger (1994): Rewrite-Based Equational Theorem Proving with Selection and Simplification. J. Log. Comput. 4(3), pp. 217-247, doi:10.1093/logcom/4.3.217.

[5] Christoph Benzmüller, Armin Fiedler, Andreas Meier, Martin Pollet \& Jörg H. Siekmann (2006): Omega. In: The Seventeen Provers of the World. Lecture Notes in Computer Science 3600, Springer, pp. 127-141, doi:10.1007/11542384_17.

[6] Marc Bezem, Dimitri Hendriks \& Hans de Nivelle (2002): Automated Proof Construction in Type Theory Using Resolution. J. Autom. Reasoning 29(3-4), pp. 253-275, doi:10.1007/1072195i_10.

[7] Anatoli Degtyarev \& Andrei Voronkov (2001): Equality Reasoning in Sequent-Based Calculi. In John Alan Robinson \& Andrei Voronkov, editors: Handbook of Automated Reasoning. Elsevier and MIT Press, pp. 611-706, doi:10.1016/B978-044450813-3/50012-6.

[8] Nachum Dershowitz (1982): Orderings for Term-Rewriting Systems. Theor. Comput. Sci. 17, pp. 279-301, doi:10.1016/0304-3975(82)90026-3.

[9] Nachum Dershowitz, Jieh Hsiang, N. Alan Josephson \& David A. Plaisted (1983): Associative-Commutative Rewriting. In: IJCAI. pp. 940-944.

[10] Gilles Dowek, Thérèse Hardin \& Claude Kirchner (2003): Theorem Proving Modulo. J. Autom. Reasoning 31(1), pp. 33-72, doi:10.1007/3-540-46508-1_1.

[11] Harald Ganzinger, Robert Nieuwenhuis \& Pilar Nivela (2004): Fast Term Indexing with Coded Context Trees. J. Autom. Reasoning 32(2), pp. 103-120, doi:10.1023/B:JARS.0000029963.64213.ac.

[12] Peter Graf (1995): Substitution Tree Indexing. In Jieh Hsiang, editor: Rewriting Techniques and Applications, 6th International Conference, RTA-95, Kaiserslautern, Germany, April 5-7, 1995, Proceedings. Lecture Notes in Computer Science 914, Springer, pp. 117-131.

[13] John Harrison (2009): Handbook of Practical Logic and Automated Reasoning. Cambridge University Press. 
[14] Joe Hurd (1999): Integrating Gandalf and HOL. In Yves Bertot, Gilles Dowek, André Hirschowitz, C. Paulin \& Laurent Théry, editors: TPHOLs. Lecture Notes in Computer Science 1690, Springer, pp. 311 322, doi:10.1007/3-540-48256-3_21. Available at http://link.springer.de/link/service/series/ 0558/bibs/1690/16900311.htm.

[15] Joe Hurd (2003): First-Order Proof Tactics in Higher-Order Logic Theorem Provers. Technical Report NASA/CP-2003-212448, Nasa technical reports.

[16] Donald Knuth \& P. Bendix (1970): Simple word problems in universal algebras. Computational problems in Abstract Algebra (J. Leech ed.), pp. 263-297.

[17] W. McCune (1992): Experiments with discrimination tree indexing and path indexing for term retrieval. Journal of Automated Reasoning 9(2), pp. 147-167, doi:10.1007/BF00245458.

[18] Jia Meng \& Lawrence C. Paulson (2008): Translating Higher-Order Clauses to First-Order Clauses. J. Autom. Reasoning 40(1), pp. 35-60, doi:10.1007/s10817-007-9085-y.

[19] Jia Meng, Claire Quigley \& Lawrence C. Paulson (2006): Automation for interactive proof: First prototype. Inf. Comput. 204(10), pp. 1575-1596, doi:10.1016/j.ic.2005.05.010.

[20] Robert Nieuwenhuis \& Alberto Rubio (2001): Paramodulation-based thorem proving. In John Alan Robinson \& Andrei Voronkov, editors: Handbook of Automated Reasoning. Elsevier and MIT Press, pp. 471-443, doi:10.1016/B978-044450813-3/50009-6. ISBN-0-262-18223-8.

[21] Lawrence C. Paulson (1999): A Generic Tableau Prover and its Integration with Isabelle. J. UCS 5(3), pp. 73-87.

[22] Alexandre Riazanov \& Andrei Voronkov (2002): The Design and Implementation of Vampire. AI Communications 15(2-3), pp. 91-110.

[23] Alexandre Riazanov \& Andrei Voronkov (2003): Limited resource strategy in resolution theorem proving. J. Symb. Comput. 36(1-2), pp. 101-115, doi:10.1016/S0747-7171(03)00040-3.

[24] Matthieu Sozeau (2009): A New Look at Generalized Rewriting in Type Theory. Journal of Formalized Reasoning 2(1), pp. 41-62.

[25] Geoff Sutcliffe (2009): The 4th IJCAR Automated Theorem Proving System Competition - CASC-J4. AI Commun. 22(1), pp. 59-72.

[26] Larry Wos, George A. Robinson, Daniel F. Carson \& Leon Shalla (1967): The Concept of Demodulation in Theorem Proving. J. ACM 14(4), pp. 698-709, doi:10.1145/321420.321429. 